\title{
Actividades exploratorio investigativas en clases de matemáticas
}

\author{
Exploratory Research Activities in Math Class
}

\author{
Atividades exploratórias em aulas de matemática
}

Leidy Johana Limas-Berrio ${ }^{a^{*}}$, Alfonso Jiménez-Espinosa ${ }^{\mathrm{b}}$

\author{
${ }^{a}$ Docente catedrática (Uptc)., Universidad Pedagógica y Tecnológica de Colombia-Colombia. \\ Orcid: 0000-0002-8307-3664 \\ ${ }^{\text {b} D o c t o r ~ Y ~ P o s d o c t o r ~ e n ~ E d u c a c i o ́ n ~ M a t e m a ́ t i c a, ~ P r o f e s o r ~ T i t u l a r ~ U n i v e r s i d a d ~ P e d a g o ́ g i c a ~ y ~ T e c n o l o ́ g i c a ~ d e ~}$ \\ Colombia - UPTC. Orcid: 0000-0001-8782-7892
}

Forma de citar: Limas, L., Jiménez, A. (2017). Actividades exploratorio investigativas en clase de matemáticas. Eco matemático 8(1). 93-105

Recibido: Octubre 28 de 2016

Aceptado: Noviembre 15 de 2016

\section{Palabras clave}

Actividades exploratorio investigativas, tareas exploratorio investigativas, investigación matemática.

\section{Keywords}

Exploratory activities, exploratory tasks, mathematical research.

\begin{abstract}
Resumen: Este artículo de revisión define las actividades exploratorio investigativas, las tareas investigativas y las aulas investigativas o investigaciones matemáticas y sus características, para lo cual comienza revisando algunos términos de uso común en el aula de clase de matemáticas, como son ejercicio, problema y situación problema; justamente para establecer diferencias con las primeras. Además se detallan los roles, tanto del docente como del estudiante en el desarrollo de este tipo de actividades; también se presenta algunas particularidades de una investigación matemática. Finalmente se muestra un amplio estado de la cuestión sobre investigaciones que se han realizado acerca de actividades exploratorio investigativas y sus contribuciones en la enseñanza y el aprendizaje de las matemáticas.
\end{abstract}

\begin{abstract}
This review article establishes the exploratory research activities, investigation tasks and study rooms or mathematical investigations, and their characteristics, in order to begin reviewing the use of common terms in the classroom, such as exercise, problem and problem solving, precisely in order to distinguish, it is made to establish some differences from the initial activities. Moreover, it highlights the student and teacher roles in the development of such activities. The article also presents some specific mathematics research. Finally, it demonstrates an open attitude to the theme in question, whilst considering the preexisting research into exploratory research activities and its contribution to the teaching and learning process.
\end{abstract}

\footnotetext{
* Autor para correspondencia limasleidy@hotmail.com

http://dx.doi.org/10.22463/17948231.1480
} 


\section{Palavras-chave}

Atividades exploratórias, tarefas exploratórias, pesquisa matemática.
Resumo: Este artigo de revisão define as atividades de exploração de pesquisa, as tarefas de pesquisa e as salas de pesquisa ou pesquisas de matemática e suas características. Assim, ele começa a verificar algumas definições comuns de uso dentro das salas de aula de matemática. Por isso, são broca, problema e a situação problemática. Na verdade, é feito para estabelecer algumas diferenças entre os primeiros. Além disso, destaca os papéis de alunos e professores no desenvolvimento dessas atividades. Além disso, apresenta algumas pesquisas de particularidades matemáticas. Finalmente, destaca-se um amplo estado do tema do qual se fala, levando em conta as pesquisas realizadas sobre as atividades de pesquisa exploratória e sua contribuição no processo de ensino e aprendizagem de matemática.

\section{Introducción}

Sin lugar a duda una de las preocupaciones de los profesores de matemáticas es despertar el interés de los estudiantes hacia esta disciplina, pues muchos la consideran poco útil por no percibir su aplicabilidad inmediata en situaciones de la vida cotidiana (Assis, 2012); a, Pabón (2014) señálalo que se puede agregar que no hay conexión entre los conocimientos adquiridos estudiados en el aula de clase y la utilidad, en situaciones del entorno. (Pabón, 2014).

Teniendo en cuenta esta problemática en el aula y con el objetivo de encontrar alternativas de solución, se habla de introducir una nueva dinámica a las clase de matemáticas, a través de actividades exploratorio investigativas; las cuales pueden proporcionar una mayor motivación e interacción entre los estudiantes, pues ambientes de esa naturaleza pueden ofrecer un contexto rico y fructífero para la negociación de significados (Bruner, 2002 citado en Assis, 2012) entre los estudiantes y entre el estudiante y el profesor. Ciompi (1999; citado en Assis, 2012) señala que la aplicación repetida de situaciones rutinarias tienden a provocar una "neutralización emocional" de un sentimiento positivo, en algunos casos transformándolo en un sentimiento negativo, ya que desarrollar la misma tarea muchas veces se torna tedioso y aburrido. Si bien es cierto nos encontramos frente a múltiples dificultades en el aula de clase y este tipo de actividades permiten una forma diferente de desarrollar una clase de matemáticas, la dinámica de la clase es diferente.
Se planean actividades abiertas donde no se pide que se encuentren resultados fijos, que se desarrollen procesos, ni mucho menos que se sigan algoritmos; sino que son actividades abiertas y desafiantes donde el alumno pone a prueba su creatividad, lanza conjeturas y las compara con las de sus colegas, para al final en plenaria encontrar la conjetura válida y más convincente; lo que hace que se les brinde trabajos relacionados a dos posibilidades muy importantes como es la exploración y la investigación (Assis, 2012). Este enfoque de trabajo del aula de matemáticas nace en Portugal y Brasil, hace cerca de veinte años y en estos países se ha venido consolidando poco a poco.

Este texto comienza destacando algunos aspectos conocidos en la clase de matemáticas, como son ejercicio, problema y situación problema. Se definen y discuten teóricamente las actividades exploratorio investigativas, las tareas y las aulas investigativas y el rol, tanto del docente como del estudiante, además se señalan las características de una investigación matemática y finalmente se cuenta con el estado del arte y la revisión de algunas investigaciones que se han desarrollado al respecto.

\section{Aspectos teóricos}

Este apartado examina perspectivas relacionadas con las actividades exploratorio-investigativas y otras de características similares, como una posibilidad real de transformar la dinámica de la clase de matemáticas. Para esto comenzamos revisando la literatura más conocida al respecto. 
Ejercicio, problema y situación problema

En el lenguaje usado en las clases de matemáticas es frecuente hablar de ejercicios, problemas y situaciones problema, o problemáticas. Con el ánimo de establecer diferencias con las actividades exploratorio investigaciones o las aulas investigativas, conceptualizamos a continuación estos tres elementos. Para empezar es necesario discutir y analizar el modo en que unas tareas se distinguen de otras, pues en el desarrollo del trabajo docente, realmente no parece haber suficiente nitidez. Es claro que cada una se refiere a actividades con características diferentes.

Pozo y Echeverría (1998) diferencian un problema de un ejercicio, señalando que en el ejercicio se disponen y se utilizan mecanismos que llevan de forma inmediata a la solución, lo que no ocurre con el problema, a pesar que es posible que el ejercicio represente un problema para una persona mientras que para otra no. El ejercicio tiene mecanismos para resolverlo con una inversión mínima de recursos cognitivos.

Para D'Amore (2006), "se tiene un ejercicio cuando la resolución prevé que se tengan que utilizar reglas y procedimientos ya aprendidos, aunque aún en vías de consolidación (...) en cambio, se utiliza un problema cuando una o más reglas o uno o más procedimientos no son todavía bagaje cognitivo del resolutor...” (p. 294). Este autor deja claro que estas no son definiciones, sino solo intentos de clarificación y que están ligados a hechos y actitudes de relación entre alumno, saber y profesor. El problema también depende del nivel en que se pretenda resolver, pues como ejemplifica el mismo autor, un ejercicio para un grado $5^{\circ}$ de educación básica puede ser un problema para un niño de grado $2^{\circ}$; pero que al ser colocado a alumnos de este nivel, las soluciones pueden ser muy creativas e ingeniosas. A este tipo de problemas, el autor los llama "ejercicios anticipados" (Ídem). Con base en esta última situación el autor aclara que no es el texto en sí lo que constituye un ejercicio o un problema, sino algo más global y ligado a cada situación didáctica, a la intención del profesor y el nivel de los estudiantes y la situación de contexto en que se presentan.

Aquí podemos cuestionar la creencia que tienen muchos profesores con respecto a que para aprender matemáticas hay que hacer muchos ejercicios; al respecto Perussi (2006) afirma que aprender matemáticas no es hacer ejercicios, sino que es ir más allá de la búsqueda de una respuesta correcta; es saber trazar un camino, si es el caso, abandonarlo y buscar otro, ser capaz de justificar sus elecciones, decisiones y acciones.

De igual manera Pabón (2014), señala que el proceso de aprendizaje no se fundamenta en la exposición de contenidos, sino que por el contrario es una experiencia en la que el estudiante pone a prueba sus capacidades, explorando y conjeturando para construir nuevos conocimientos, así mismo Ángulo (2006, citado en Pacheco; 2016) afirma que enseñar matemáticas es proporcionar medios de reflexión, pues expresa que es una ciencia que debe ir adaptándose a los cambios continuos.

En el aula de clase de matemáticas predomina la realización de ejercicios y en mucha menor medida los problemas; según Priscila (2011) estos tienen una característica en común; pues el profesor en la mayoría de los casos sabe de antemano cuál es la respuesta a la cual deben llegar los estudiantes, y cuál es el camino que deben tomar para encontrar la solución válida; en ambos casos el enunciado indica claramente lo que se da y lo que se pide.

En el salón de clase puede haber otro tipo de actividad, diferente a las dos anteriores; nos referimos a la situación problema, la cual puede ser más provechosa que las dos anteriores, ya que le permite al estudiante buscar herramientas propias para trazar el camino y encontrar la solución. Para D'Amore, una situación problema puede describirse como "una situación de aprendizaje concebida de manera tal que los estudiantes no pueden resolver la cuestión por simple repetición $o$ aplicación de conocimientos o competencias adquiridas, sino que se necesita la formulación de nuevas hipótesis” (p. 295). 
Para este autor, las situaciones problémicas entran en otra dinámica ya que es diferente a la de los ejercicios y problemas; se trata de otro enfoque didáctico que él llama de la emancipación y el asombro, en las que el alumno se apoya en su creatividad. Para vincular a un estudiante a este tipo de actividad, la motivación debe ser fuerte y estimulante, pues de no serlo puede convertirse en un obstáculo. D'Amore destaca que crear situaciones problema no es una tarea fácil y requiere creatividad, tiempo y energía, para lo cual se debe estar plenamente convencido y tener un proyecto claramente delineado, además de ser consciente de las operaciones mentales requeridas en la solución de la situación planteada. Las situaciones problemáticas requieren "que la actividad se halle fuertemente estructurada, prevista en todos sus detalles metodológicos y de contenido matemático (...) para el estudiante debe sentirse libre de usar sus propios recursos mentales, independientemente de sus conocimientos previos" (p. 296).

\section{Actividades exploratorio- investigativas}

Fiorentini y Christopher (2006; citado en Assis, 2012) definen las prácticas de exploración e investigación como una opción para problematizar y promover significados para la matemática escolar, pues generalmente permiten construir conceptos a través de la aplicación de este tipo de actividad. Las actividades exploratorio investigativas se pueden considerar como una herramienta que permite desarrollar habilidades y capacidades mentales. Según Miranda y Pereira (2014) las actividades exploratoria investigativas son una opción interesante para investigar durante el proceso de desarrollo de las tareas, ya que aquí se requiere formular preguntas, organizar ideas, plantear conjeturas y poder probarlas.

Las actividades de naturaleza exploratoria e investigativa se pueden considerar como una manera de trabajar con los estudiantes en el aula de clase, donde la característica fundamental de estas actividades es la motivación y el desafío que les proporciona; además facilitar actividades investigativas en el aula, genera desequilibrios que llevan al raciocinio del alumno. Juliani (2007) señala que estas actividades movilizan múltiples significados que hacen que se despierte la curiosidad y el interés de los alumnos por el estudio de la matemática.

Siendo estas actividades situaciones en las que se sabe el punto de partida, pero no el punto de llegada (Ponte, 2003), es importante que el docente analice los caminos que pueden surgir en el estudiante a la hora de enfrentarse a una situación; de esta manera podrá brindar a los estudiantes una buena orientación en la configuración y discusión de resultados, permitiendo que cada uno defienda sus diferentes puntos de vista y respete el de los demás.

Según Costa y Silva (2015, p. 9) las actividades de investigación incluyen elementos básicos como:

1. Movilización: la acción que tiene como objetivo movilizar a los estudiantes a participar en la clase y a partir de esa participación, aprender.

2. Aprendizaje: buscar sobre el aprendizaje de los estudiantes y entender cómo comprenden ese aprender.

3. Investigación: práctica de la enseñanza basada en el dialogo y en la valorización de las acciones investigativas de los estudiantes en el aula de clase.

Fiorentini y Cristóvão (2006) señalan que las actividades exploratorio investigativas movilizan múltiples significados de un concepto, lo que hacen que los estudiantes durante su desarrollo se muestren más activos y más curiosos; por tanto estas actividades son esenciales para problematizar y producir significados de un concepto matemático. En el desarrollo de actividades exploratorio investigativas puede que los estudiantes se sientan perdidos por la falta de experiencia con este tipo de trabajos; pero poco a poco comienzan a darse cuenta que pueden formular preguntas que le ayudan a descubrir y encontrar maneras interesantes para resolver una situación de manera intuitiva y validar sus observaciones.Las 
actividades exploratorio- investigativas brindan a los estudiantes la oportunidad de participar en un juego intelectual de sus propios conocimientos, es por esta razón que la comunicación se hace importante para expresar las ideas en grupos. En el desarrollo de este tipo de actividades algunas decisiones deben ser tomadas por el profesor; como el tiempo, la selección de la situación, la forma de trabajo, entre otras, y otras decisiones son negociadas con los estudiantes.

\section{Tareas exploratorio- investigativas}

Sanches \& Beline (2013) señalan que las tareas exploratorio- investigativas generalmente son abiertas y poco estructuradas; permiten al alumno explorar, elaborar sus propias preguntas y conjeturas, y buscar la argumentación para la justificación y validación de éstas.

Ponte (2003) establece que las tareas exploratorioinvestigativastienen cuatro dimensiones básicas: el grado de dificultad, el contexto de referencia, la estructura y el tiempo requerido para la resolución; indicando de este modo que las tareas exploratorio investigativas son de estructura abierta, y con un alto grado de dificultad. El autor señala que los problemas son tareas cerradas con dificultad alta, los ejercicios son tareas sin gran dificultad y de estructura cerrada y la investigación matemática tiene un alto grado de dificultad y con estructura abierta.

Ernest (1998; citado en Miranda y Pereira, 2014) concibe una tarea exploratorio- investigativa como "una situación en la cual un individuo o un grupo es llamado a realizar una tarea para la cual no hay un algoritmo fácilmente accesible que determine completamente el método de solución" (p. 12); es decir; este tipo de tareas se describen por tener un carácter amplio, desafiador y admitir muchas respuestas que tienen como punto de partida un enunciado, un propósito y objetivos poco precisos que hacen que los mismos estudiantes tengan que trazar sus caminos y buscar argumentos.

Por otro lado Miranda y Pereira (2014) consideran que las tareas exploratorio-investigativas son el punto de partida para los debates en matemáticas, ya sea sobre la resolución de problemas o sobre las investigaciones.

Assis, Godino y Frade (2012) identifican patrones que delinean el escenario de las tareas exploratorias investigativas; algunos de ellos son: los alumnos deben asumir la responsabilidad de resolución de la tarea, el profesor debe llevar a los estudiante a construir el entendimiento, y el docente debe proporcionar información sin dar la solución al problema; así mismo debe propiciar situaciones en las que ellos se dediquen al descubrimiento.Además es necesario hacer que los estudiantes tomen nota de cada proceso realizado durante el desarrollo de las actividades; pues en este caso, el recuento no es un proceso valido, este es considerado como un poco sofisticado en matemáticas.

Es claro que el aprendizaje de las matemáticas depende mucho de las tareas propuestas por el profesor, pues según Ponte (2003) las características de una tarea son relativas a la persona que las realiza, pues una misma situación puede ser para una persona un ejercicio y para otra un problema. El autor señala que en la realización de tareas exploratorio investigativas en el aula, uno de los momentos más importantes para la institucionalización de los aprendizajes es la discusión final; pues es aquí donde se da a conocer las exploraciones y los caminos que toma cada estudiante.

\section{Aula investigativa}

Al respecto Facanali (2004) afirma que "un aula investigativa es aquella que privilegia la realización de investigaciones matemáticas en el aula de clase por los estudiantes" (p. 39); es decir, estas asumen la participación de los alumnos con tareas investigativas que les permita realizar actividad matemática para luego ejecutar la realización de investigaciones matemáticas; por otro lado la autora reconoce las aulas investigativas "como un escenario propicio para la reflexión y producción de saberes, no solamente por parte de los alumnos, sino también y principalmente por 
parte de los profesores" (p. 42). Esto debido a que los profesores deben hacer una buena preparación previa de cada actividad, además durante el desarrollo de la actividad debe estar estudiando, reflexionando y (re)significando saberes en la interacción con los estudiantes, pues en este tipo de actividades los profesores no saben de antemano las respuestas y tampoco puede prever las exploraciones que realizarán los estudiantes en cada situación.

Una clase con enfoque investigativo es aquella en la que los alumnos participan en tareas investigativas. Ponte, Fonseca y Brunheira, describen las fases de una clase de investigación, con "introducción de la tarea, desarrollo del trabajo y discusión final o reflexión”; y afirman además que "Una aula investigativa es aquella que supone el envolvimiento de los alumnos en tareas investigativas, y por tanto, <ayuda a trazar en el aula de clase un espíritu de actividad matemática genuina>" (Ponte, Brocado e Oliveira, 2006, citado en Assis 2012 p. 59).

Monteiro y Gonçalves (2011) señalan que el propósito de trabajar con aulas investigativas es desarrollar en los estudiantes el hábito de buscar la validez de un proceso, pues esto los obliga a construir argumentos y aprender a comunicarse.

Según Priscila (2011) estas actividades han ganado mucha visibilidad en los planes de estudio como una alternativa didáctico pedagógica para la enseñanza de las matemáticas; de otro lado Souza y Cristovão (2010) manifiestan que trabajar bajo el enfoque de aulas investigativas brinda al estudiante la oportunidad de ser protagonista de su propio aprendizaje y de la misma manera la clase se hace más significativa para ellos.

En un clase investigativa o con enfoque exploratorio investigativa, la primea fase consiste en explicar la tarea y el tipo de trabajo que se desarrolla en la investigación, puede ser de manera escrita u oral o una parte escrita y otra oral; en la fase de desarrollo del trabajo se pretende que los estudiantes desarrollen actitud de investigador, tratando de enfocar la clase en la actividad de los alumnos, sus ideas, sus aportes y sus inquietudes con respecto a la temática; para esto el profesor debe tener una actitud cuestionadora ante las peticiones y preguntas de los estudiantes; por último la reflexión permite al estudiante valorar los procesos realizados incluso cuando no llegan al resultado esperado.

Un aula investigativa requiere de la participación activa de los estudiantes, pues según Souza y Cristovão (2010) estas consisten en tareas abiertas, donde los estudiantes tienen la oportunidad de elegir el camino para llegar a la construcción de su conocimiento; en estas, en ocasiones el papel se invierte; es decir, los profesores aprenden y los estudiantes enseñan, pero no solamente es una relación entre estudiante y profesor sino que implica a toda la comunidad educativa.

Según Sánchez y Beline (2013) el aula investigativa se estructura en tres etapas: introducir la tarea exploratorio-investigativa, desenvolver la investigación matemática, y la discusión de los resultados encontrados por los investigadores, en este caso los alumnos. Ponte (2010) establece tres fases de una clase exploratoria investigativa, así; introducción, desarrollo del trabajo y discusión de resultados. En la primera fase se explica cómo interpretar la formulación del trabajo y cuál es la forma de llevarlo a cabo; en la segunda los estudiantes deben trabajar autónomamente, sea de manera individual o de manera colectiva según las indicaciones del docente y por último en la discusión de resultados comparten ideas generadas por cada estudiante o por cada grupo, con el fin de plasmar o institucionalizar un nuevo conocimiento matemático. Es de destacar que esa etapa de trabajo del estudiante debe hacerse en dos momentos: primero de forma individual, lo cual permite que cada uno proponga sus soluciones y luego en pequeños grupos de tres estudiantes se confrontan las primeras soluciones y la consistencia de los mismos, antes de llevar los resultados a la plenaria (Jiménez, et al, 2010). De esta forma, la tercera fase es la de la validación grupal de las conjeturas 
y soluciones; es decir, de la institucionalización del saber matemático construido.

Santos (2011) señala que la preparación de un aula investigativa es una etapa importante; en esta se debe partir por definir los objetivos, siendo creativos y teniendo en cuenta aspectos como edad, familiaridad que tienen los estudiantes con el tema, conocimientos previos e intereses. El autor señala que en la etapa de la discusión final además de una buena preparación matemática por parte del profesor se necesita de capacidad de gestión de la dinámica colectiva; el docente en esta etapa tiene la función de moderador y orientador con el fin de estimular la comunicación entre los alumnos explicando sus ideas y conclusiones.

\section{Rol del profesor en las aulas investigativas}

Pacheco (2016) señala que el papel del profesor es fundamental en el aula de clases, ya que mediante su buena actitud y disposición logra estimular a los estudiantes, además mantiene de mantener un buen ambiente de trabajo. En el desarrollo de las actividades exploratorio investigativas es importante que el profesor tenga buenas razones para hacer que los estudiantes se involucren, sean atraídos por las diferentes actividades propuestas en el aula; pues como señala Skovsmose (2000) un escenario para la investigación solo se constituye cuando se convierte en una postura investigativa y es aceptada la invitación por los estudiantes. Aquí el profesor tiene el papel de liderar permanentemente la comunicación en el aula, pues según Ponte (2010), si el docente no responde inquietudes de los estudiantes, estos pueden perder la motivación en el desarrollo de la actividad; por otro lado, si proporciona la repuesta a los alumnos se termina el trabajo por parte de ellos y se pierde el beneficio en el aprendizaje del estudiante. Además de esto debe haber una mayor preparación por parte del profesor pues es imposible que él pueda prever todas las preguntas y sugerencias que surgen en el estudiante durante este proceso.

Oliveira, Segurado y Ponte (1996) indican que el profesor tiene un papel fundamental en la planificación de actividades de investigación en el aula; pues este enfrenta decisiones difíciles en cuanto a la gestión y al tiempo; además durante este proceso debe generar preguntas que estimulen e inviten a los estudiantes a mirar en otras direcciones y a reflexionar.

\section{Papel de estudiante en las aulas investigativas}

Cuando los estudiantes trabajan en grupos lo hacen más activamente y se involucran más, tratado de dar sentido a la situación y por tanto tratando de interpretar situaciones para reestructurar lo que ya saben. Ponte (2003) señala que el docente tiene un papel muy importante en el desarrollo de este tipo de actividades; pues el éxito depende del modo en que el profesor responda a las dudas de los estudiantes, pues se trata de estimularlos sin darles directamente la respuesta, además de fomentar la formulación de nuevas preguntas.

Según Bishop y Goffree (1986; citado en ponte, 2010) los estudiantes deben ser motivados a compartir ideas con sus colegas, por lo que el estado de ánimo del alumno es primordial en el desarrollo de este tipo de actividades. El docente además de apoyar el trabajo de los estudiantes y promover las reflexiones por medio de información que haga que el trabajo fluya, debe desafiar a los estudiantes y evaluar su progreso.

De la misma manera Ponte, Brocardo y Oliveira (2006; citado en Martendal y Belline, 2013) indican que el docente debe dar autonomía a los estudiantes, garantizar que el trabajo fluya $\mathrm{y}$ que sea significativo, realizando preguntas y pidiendo explicaciones tratando de comprender el pensamiento de los alumnos; además, debe evitar dar opiniones muy concretas.

\section{Investigaciones matemáticas}

En el desarrollo del trabajo docente cada vez es más importante que el profesor tenga conocimientos básicos de investigación e intente investigar su propia práctica; además de incentivar y promover esta habilidad en sus alumnos desde su propia aula 
de clase (Jiménez, 2002). Pirie (1987; citado en Brocardo, 2001) señala que investigar se trata de una situación abierta cuya exploración no tiene como objetivo llegar a una respuesta correcta, pues "el objetivo es el viaje, no el destino" (p.98); es decir, se trata de comprender y descubrir relaciones y patrones, aprendiendo a medida que busca apoderarse de una situación que lo va llevando a sentir un gran desafío.

Ponte (2003) señala que investigar no es más que buscar conocer, buscar comprender, buscar y encontrar solución a los problemas; además indica que es una capacidad importante para todos los ciudadanos, y por tanto vital en el trabajo tanto del docente como del estudiante. Este autor señala que la investigación involucra esencialmente tres actividades: estudiar, conversar y escribir. La primera hace referencia a estudiar autores clásicos y modernos del área de la matemática y de la educación matemática sobre las temáticas de la que se esté trabajando; la segunda hace referencia a hablar con personalidades que sepan de la temática, con el fin de intercambiar ideas y comprender sus puntos de vista; por último plasmar las ideas, experiencias, deseos y frustraciones con el objetivo de que sean conocidas por la comunidad profesional.

Ponte (2010) señala que investigar en matemáticas incluye la formulación de peguntas, las cuales cambian a medida que avanza el trabajo. Además indica que esta actividad implica producción, análisis, demostración y comunicación de los resultados ya sea en matemáticas o en otra ciencia.

Oliveira, Segurado y Ponte (1996) señalan que las investigaciones matemáticas designan un tipo de actividad centrada en procesos matemáticos, tales como buscar regularidades, formular, probar, justificar, reflexionar y generalizar. Estas se caracterizan por el estímulo que proporciona al alumno el justificar sus afirmaciones y la explicación matemática ante sus compañeros.

Por otro lado, Monteiro y Gonçalves (2011) indican que "una investigación matemática es un proceso que proporciona al alumno la construcción de conocimiento de una manera no sistemática" ( $p$. $2)$; es decir, el estudiante hace inferencias que conducen a la solución y estas se pueden diversificar en el proceso; por esta razón estas actividades requieren más habilidad por parte de los docentes. Así mismo revelan que los estudiantes realizan investigaciones en el momento en que reflexionan sobre las posibilidades, sobre los caminos y las formas de construcción del proceso de solución; en este caso es el estudiante quien toma la decisión sobre el método de exploración y decide la manera en que quiere aprovechar cierta situación.

Ponte (2003) señala que investigar no necesariamente es tratar con problemas de gran dificultad, significa trabajar a partir de cuestiones que nos interesan y que se presentan de manera confusa; al hablar de investigación matemática el alumno parte de una cuestión general poco estructurada e intenta formular algo más específico y sobre esto producir varias conjeturas que deben ser probadas y argumentadas o descartadas. De otra parte, para Sánchez y Beline (2013) la investigación estimula a los alumnos a utilizar los conocimientos ya vistos en el aula de clase, permitiendo una mejor apropiación de los objetos de estudio; por esto las investigaciones matemáticas pueden de alguna manera desarrollar en los estudiantes habilidades como conjeturar, justificar, argumentar y deducir. Para Juliani (2007) también las investigaciones se constituyen en una estrategia pedagógica que moviliza otras formas de aprendizaje en los alumnos.

Las investigaciones matemáticas son consideradas situaciones problema, desafiadoras y abiertas que permiten libertad al estudiante al momento de desarrollarla; por tanto, estas situaciones en el aula de clase ayudan a los maestros a identificar las dificultades de los alumnos, contribuyendo así al desarrollo del pensamiento e identificando la secuencia de ciertas regularidades (Sánchez y Beline, 2013).

Otros autores como Perussi (2006) no hablan de 
investigaciones matemáticas, sino de actividades investigativas, y las caracterizan como una herramienta valiosa para la enseñanza de las matemáticas, ya que despierta en el estudiante el espíritu investigativo y contribuyen al desarrollo de diferentes habilidades; siendo esto lo que se quiere en las aulas de clase, que los estudiantes sean autónomos de su proceso educativo, por lo que el profesor debe ser un mediador esencial en este tipo de actividades.

Ponte, Brocardo y Oliveira (2003) señalan que para que el alumno pueda investigar es necesario que trabaje de forma autónoma; es por esto que el profesor debe tomar un papel de regulador de la actividad. Además es necesario que el profesor valore la realización, evaluación y discusión de actividades de investigación con el fin de que sea una actividad rica en matemáticas para los estudiantes, pues en la mayoría de los casos el docente sabe y espera con anterioridad el camino que deben tomar los estudiantes, rechazando otras posibilidades llevadas a cabo por ellos. Cuando se rechazan de plano otras interpretaciones de los alumnos sin dar argumentos, conlleva a la falta de sentido de la matemática para los estudiantes y a la poca participación en la construcción y (re) significación de sus saberes (Jiménez, 2002); pero para que esto suceda, se requiere que la actividad de construcción del conocimiento esté relacionada con la realidad.

Otra característica de las investigaciones matemáticas es que el alumno es llamado a actuar como un pequeño matemático, no solamente en la realización de pruebas y conjeturas sino en la presentación de resultados, en la argumentación y en la discusión de estos frente a sus compañeros y al profesor (Ponte, Brocardo y Oliveira, 2003).

En el contexto discutido en este texto, se usan con frecuencia términos que aparentemente son similares, el de tarea y el de actividad, pero realmente se refieren a cosas diferentes (Christiansen y Walther, 1986; citados en Brocardo, 2001). La actividad se refiere esencialmente al alumno, a aquello que hace en un determinado contexto; la tarea representa el objetivo de cada una de las acciones llevadas a cabo por los estudiantes; la tarea aunque puede ser definida por el alumno es generalmente propuesta por el profesor, pues ésta en sí no contiene conceptos ni estructuras matemáticas.

Es importante que a partir de las experiencias que tienen los estudiantes se defina el grado de dificultad de las tareas; pues cuanto más estructurada sea ésta, más significativa va a ser para los alumnos (Porfirio y Oliveira, 1999; citados en Brocardo, 2001). Así por ejemplo, una propuesta muy abierta puede parecer muy vaga y hacer que los estudiantes no se sienten desafiados para comenzar cualquier exploración.

Según Ernest (1996, citado en Assis, Godino y Frade, 2012) el objetivo de una investigación matemática es explorar todos los caminos a partir de una situación; esta se considera un proceso divergente pues se conoce el punto de partida mas no el punto de llegada, así como tampoco se conocen los caminos para llegar a ese punto de partida.

La investigación como metodología implica la participación de los alumnos y estimula la participación entre ellos; además como lo señala Santos (2011) provoca la capacidad de raciocinio, posibilita el empleo de conceptos matemáticos para desarrollar actividades propuestas y contribuye a la mejora del aprendizaje del alumno, permitiendo un dialogo entre alumnos y entre los alumnos y el profesor; para que esto sea posible es fundamental la colaboración, la cooperación y el compromiso tanto del profesor como de los estudiantes.

Según Ponte (2003) las investigaciones y las exploraciones matemáticas son actividades diferentes; las primeras son situaciones problemáticas desafiantes y abiertas que permite al estudiante tomar diversas alternativas de solución, y las exploraciones tienden a ser más libres y menos sistemáticas. 


\section{Estado}

En Portugal, Ponte $(1990,1992)$ ha realizado varias investigaciones sobre la dinámica de las aulas a través de las clases con enfoque exploratorio-investigativas. Los resultados muestran que la dinámica de la clase se transforma, que los estudiantes se envuelven en la actividad matemática y que los alumnos comienzan a pensar como verdaderos matemáticos. De la misma forma en Brasil, Oliveira e Fiorentini (2009) en una investigación sobre la construcción de los números irracionales, muestra que los alumnos realmente construyen el concepto y que incluso llegan a resultados, que para su nivel parecen sorprendentes

Assis (2012) en su investigación hace un análisis para identificar los aspectos constitutivos de producción de significados matemáticos en contexto de aulas exploratorio investigativas y evaluar la idoneidad del proceso didáctico vivido. Este análisis se centra en la enseñanza y el aprendizaje de un grupo de alumnos de grado séptimo de una escuela pública de Belo Horizonte, con la realización de actividades exploratorio investigativas implementadas en clases de matemáticas. Esta investigación fue desarrollada bajo un enfoque cualitativo, utilizando como herramientas de recolección de información transcripciones de grabaciones de audio, videos de los procesos vividos y diarios de campo generados por los estudiantes en cada fase. Esta investigación mostró que las actividades exploratorio- investigativas en el aula son fuertemente condicionadas por los siguientes aspectos: afectividad (normas y metanormas), actitudes, emociones, motivación, materialidad (disponibilidad de tiempo, medios, etc.), forma de interacción, tarea propuesta y familiaridad de los estudiantes con ese tipo de actividad. En este caso los alumnos no habían tenido contacto con este tipo de actividades, por lo que necesitaron gran soporte por parte del profesor, pues no basta con ofrecer situaciones ricas o crear espacios donde los alumnos tengan que argumentar y expresar lo que piensan; es necesario que el profesor cuestione y problematice las ideas de los estudiantes para vencer las dificultades y proseguir; de esta manera se puede proporcionar un contexto adecuado para la producción de significados.

Perussi (2006) realizó una investigación con el fin de conocer cómo tres estudiantes de grado octavo participan en actividades de investigación matemática y analizar la interacción con el profesor en el desarrollo de estas, mostrando un gran interés y persistencia por parte de los estudiantes y por tanto una mayor implicación en la tarea, el cambio de actitud y el liderazgo en el trabajo en grupo. Además señala que la naturaleza de las actividades de investigación puede ser la oportunidad para que los estudiantes aprendan a observar, expresar sus ideas, justificar y ampliar conocimientos en esta disciplina, pues es necesario proponer tareas en diferentes contextos a los estudiantes.

Igualmente Brocardo (2001) en su indagación muestra que la exploración continua de investigaciones matemáticas de aula son experiencias que motivan a los alumnos, ayuda a establecer un ambiente en el que participan activamente $\mathrm{y}$ facilita la comprensión de los procesos e ideas matemáticas; por tal razón los estudiantes prefieren un contexto en el que tengan un papel activo para mostrar una mayor comprensión de la naturaleza de las matemáticas. Este estudio sugiere además que es posible explorar una gran diversidad de temas matemáticos usando una metodología de investigación, donde se hace que los estudiantes realicen informes escritos de las investigaciones que realicen en el aula de clase. Afirman también que el uso de materiales de manipulación es importante para apoyar su progreso; por esto el ejercicio de escribir debe empezar en los primeros años de escolaridad con las justificaciones, los razonamientos y las explicaciones de los procesos realizados.

Por otro lado Facanali (2004) en la investigación sobre su propia práctica, en un contexto de aulas investigativas en matemáticas, con el objetivo de 
analizar el papel desempeñado por la actividad de investigaciones matemáticas en sus prácticas de aula, en su proceso de formación como profesora de matemáticas, mostró que la experiencia de planear, vivenciar, analizar, reflexionar y escribir sobre la experiencia pedagógica de trabajar con el enfoque de investigaciones matemáticas en el aula era muy formativa para el profesor investigador.

Molina (2007) en su investigación realizada en los grados quinto y sexto de educación básica con jóvenes y adultos de una escuela de la red pública de Itatiba (Brasil), con el objetivo de analizar el movimiento y la producción de conocimientos matemáticos generados en la realización de tareas exploratorio investigativas de contenidos matemáticos, concluyó que el contexto de las tareas exploratorio investigativas en el aula de educación de jóvenes y adultos, admite que los jóvenes y adultos tengan voz, expresen sus ideas y tengan pensamientos matemáticos que promueven el desarrollo de la autonomía intelectual y crítica. Esta investigación de naturaleza cualitativa, cuyos instrumentos de recolección de información fueron entrevistas semiestructuradas, grabaciones de audio de las discusiones en el aula, diarios y notas de campo recopilados por el profesor- investigador, informes individuales sobre las clases y escritos de algunos estudiantes; permitió verificarlos aportes de esta metodología para la enseñanza de las matemáticas, así como de la formación profesional y personal del profesor investigador. Además, señala que las tareas exploratorio-investigativas deben involucrar problemas abiertos en los que los estudiantes se atrevan a lanzar conjeturas y busquen la validación de éstas; la riqueza de este tipo de tareas radica en la variedad de estrategias, la posibilidad de implementar procesos de argumentación, validación y comunicación de ideas que surgen en el desarrollo del trabajo investigativo

En la misma área, Miranda y Pereira (2014) realizaron un estudio de naturaleza cualitativa, con elfin de verificarque las actividades de investigación hacen posible que los estudiantes relacionen conceptos matemáticos ya estudiados e iniciar así la construcción de otros conceptos. Así mismo, explorar el potencial de las tareas de investigación con tareas matemáticas más abiertas, son una herramienta para que los estudiantes expresen sus propias ideas e hipótesis y defiendan con argumentos lógicos y racionales sus conclusiones. En estas condiciones los estudiantes se sienten haciendo matemática y actuando como verdaderos matemáticos, desarrollan una actitud crítica acerca de las propuestas presentadas por ellos mismos, para permitir así un intercambio de experiencias y conceptos matemáticos. En esta investigación los datos fueron recolectados a través de cuestionarios respondidos por los alumnos y profesores, registro del investigador en su diario de clase, tareas escritas realizadas por los alumnos y grabaciones en audio de las clases orientadas por el maestro. Este estudio determinó que la naturaleza de las tareas de investigación proporciona retos para los estudiantes y despiertan la curiosidad matemática en ellos; además de esto pone a los estudiantes en la posición de matemáticos jóvenes haciendo y creando matemáticas. Según estos autores, así mismo estas actividades tienen el potencial de despertar la creatividad en los jóvenes para resolver situaciones inesperadas que se producen en la vida diaria, por tanto es importante preparar a los jóvenes para actuar como ciudadanos críticos para entrar al mercado del trabajo profesional (Ídem).

De igual manera Belluco (2015) en su tesis de doctorado hizo una revisión sobre la argumentación matemática con la intención de obtener elementos para la construcción de un instrumento de análisis de situaciones de enseñanza y aprendizaje en la física. Con base en esto se elaboró una secuencia de enseñanza con enfoque investigativo la cual fue aplicada a un grupo de primer año de enseñanza media de una escuela pública del estado de Santa Catarina (Brasil) con la ayuda del software videograph. Esta investigación mostró la necesidad de considerar los diferentes lenguajes usadas en la comunicación y de observar la calidad del proceso 
argumentativo en su forma y contenido. Agrega que es importante fomentar en los estudiantes la construcción de argumentos en actividades de enseñanza con enfoque investigativo para que relacionen teórica y práctica, y de esta manera logar una buena calidad del proceso argumentativo en la construcción de significados.

Se puede concluir que los argumentos que justifican la utilización de actividades exploratorio investigativas, tareas investigativas y aulas investigativas en la clase de matemáticas son evidentes y significativas, ya que contribuyen en el desarrollo mental del estudiante, mejorando la capacidad de argumentar, comprender, probar y justificar una situación matemática, pero además, y algo bien importante, generan la buena disposición de los alumnos al trabajo en matemáticas.

\section{Conclusión}

Todas las pruebas que realizan, tanto el Estado como otras instituciones a los aprendizajes de los niños y jóvenes, dejan ver que la calidad de aquellos no son lo mejor, que en gran medida el peso de la tradición pedagógica y didáctica de los profesores de matemáticas no ha evolucionado lo suficiente y que la necesidad de cambiar la dinámica de las aulas de clase es imperiosa. Una alternativa viable es la de trabajar en la clase de matemáticas las actividades exploratorio - investigativas, las tareas investigativas y las investigaciones matemáticas. Este enfoque nace en Portugal y Brasil, hace cerca de veinte años y en estos países se ha venido consolidando poco a poco. Este texto da elementos teóricos esenciales de este tipo de trabajo en el aula y da evidencias de cómo la clase de matemáticas, bajo este enfoque, realmente encuentra una forma de transformarse. Trabajar en clase bajo este enfoque invierte el orden de la misma, pues ésta no inicia, como es costumbre, con la teoría (definiciones y reglas o algoritmos) para luego ir a los ejemplos y problemas; si no por el contrario, comienza con actividades y tareas en contexto, pasa por la conjeturación y termina por la consolidación o institucionalización de los conceptos. Este es un enfoque promisorio que ha venido consolidándose y con seguridad traerá mejores aprendizajes de la matemática en niños y jóvenes.

\section{Referencias}

Assis, A. (2012 ). A produção de significados matemáticos em um contexto de aulas exploratórioinvestigativas. Belo Horizonte.

Assis, A., Godino, J. D., \& Frade, C. (2012). AS DIMENSÕES NORMATIVA E METANORMATIVA EM UM CONTEXTO DE AULAS EXPLORATÓRIO-INVESTIGATIVAS. Revista Latinoamericana de Investigación en Matemática Educativa, 171-198.

Bellucco, A. (2015 ). Argumentação matemática em aulas investigativas de fisica. Sao Pablo.

Brocardo, J. (2001). AS INVESTIGAÇÕES NA AULA DE MATEMÁTICA: UM PROJECTO CURRICULAR NO $8^{\circ}$ ANO. Lisboa.

Costa, M, \& Silva, A (2015). Aulas investigativas e a construção de conceitos de matemática: um estudo a partir da teoria de Piaget. 240-248.

D'Amore, B. (2006). Didáctica de la matemática. Bogotá: Magisterio.

Facanali, J. (2004). Um estudo sobre a própria prática em um contexto de aulas investigativas de Matemática. Campinas.

Fiorentini, D. y Cristóvão, E. (2006.). Histórias e Investigações delem Aulas de Matemática.

Jiménez, A. (2002). Quando professores de Matemática da escola e da universidade se encontram: (re) significação e reciprocidade de saberes. Tese de doutorado. Campinas (São Paulo- Brasil): FE/ Universidade Estadual de Campinas (UNICAMP). Diretor: Professor Dr. Dario Fiorentini.

Juliani, M. (2007). MINHA PRIMEIRA EXPERIÊNCIA EM AULA INVESTIGATIVA DE MATEMÁTICA.

Martendal, C. H., \& Belline, W. (2013). INVESTIGAÇÕES MATEMÁTICAS EM SALA DE AULA: PROPONDO E ANALISANDO A 


\section{APLICAÇÃO DE TAREFAS INVESTIGATIVAS} NA EDUCAÇÃO BÁSICA.

Miranda, M., \& Pereira, M. (2014). ATIVIDADES DE INVESTIGAÇÃO MATEMÁTICA. Itapina.

Molina, A. (2007). Aulas investigativas na educação de jovens e adultos (eja): o movimento de mobilizar-se e. Itatiba.

Monteiro, R., \& Gonçalves, R. H. (2011 ). Aulas Investigativas e a formação do professor de Matemática. XIII CIAEM-IACME.

Oliveira, H., Segurado, M., \& Ponte, J. P. (1996). Explorar, Investigar e Discutir na Aula de Matemática. Actas do ProfMat96, 207-213.

Oliveira, T., \& Fiorentini, D. (2009). Explorando $o$ sentido de número irracional no ensino fundamental. Pp. 131 - 148. In: Histórias de colaboração e investigações na prática pedagógica em matemáticas: ultrapassando os limites de sala de aula. 1 Ed. Campinas: Alina, Vol. 1.

Pabón, J. A. (2014 ). Las Tics y la lúdica como herramientas facilitadoras en el aprendizaje de la matemática. Revista Eco Matemático,Vol. 5 \# 1 p. 37- 48.

Pacheco, N.C. (2016). La motivación y las matemáticas. Revista Eco Matemático. Vol 7 \# 1, p. 149- 158

Perussi, R. (2006). TAREFAS INVESTIGATIVAS DE MATEMÁTICA: UMA ANÁLISE DE TRÊS ALUNAS DE $8^{a}$ SÉRIE DO ENSINO FUNDAMENTAL. Curitiba.

Ponte, J. (1990). "Las creencias y concepciones de maestros como un tema fundamental en la formación de maestros". Universidad de Lisboa. Portugal. En In K. Krainer \& F. Goffree (Eds.) On research in teacher education: From a study of teaching practices to issues in teacher education. pp.43-50.

Ponte, J. (1992). Concepções dos Professores de Matemática e Processos de Formação. Em J. P. Ponte (Ed.), Educação matemática: Temas de investigação (Pp. 185-239). Lisboa: Instituto de Inovação Educacional.

Ponte, J. P. (2003). Investigar, ensinar e aprender. 25-
Ponte, J. P. (2010 ). Explorar e Investigar em Matemática:UmaActividade Fundamental noEnsino e na Aprendizagem. REVISTA IBEROAMERICANA DE EDUCACIÓN MATEMÁTICA, 13-30.

Ponte, J. P., Brocardo, J., \& Oliveira, H. (2003 ). Investigações matemáticas na sala de aula. Belo Horizonte : Autentica editora.

Ponte, J. P., Fonseca, H., \& Brunheira, L. (1999). As actividades de investigação, o professor e a aula de Matemática. Departamento de Educação, F.C.U.L.

Pozo, J., \& Echevería, M. (1998 ). Aprender a resolver problemas e resolver problemas para aprender.

Priscila, J. (2011). INVESTIGAÇÃO MATEMÁTICA EM SALA DE AULA: AS MUDANÇASPROCEDIMENTAIS DE ALUNOS DO ENSINO MÉDIO. Actas del 3 congreso uruguayo de educación matemática.

Sanches, E, \& Beline, W. (2013). Tarefas investigativas promovendo o pensamento matemático em alunos da educação básica.

Santos, D. (2011). INVESTIGAÇÕES MATEMÁTICAS. Educação Matemática da UFOP.

Skovsmose, O. (2000). Cenários para investigação. 6691.

Souza, K., \& Cristovão, E. (2010 ). INVESTIGAÇÃO MATEMÁTICA DIFICULDADES ENCONTRADAS POR UMA PROFESSORA INICIANTE. Cadernos da Pedagogia. São Carlos, 125- 134. 